\title{
SOBRE LA PRESENCIA DE ALGUNAS ESPECIES DE ALGAS TROPICALES EN EL LITORAL DEL SURESTE IBERICO
}

\author{
J. SOTO \& F. CONDE
}

RESUMEN: Se comenta la presencia de las especies Dipterosiphonia dendritica (Ag.) Schmitz in Engl. et Prantl, Liagora farinosa Lamouroux, Polystrata fosliei (Weber van Bosse) Denizot y Pocockiella variegata (Lamourux) Papenfuss halladas en varias localidades del SE de la Peninsula Ibérica comentando su ecologia, corologia y ciertos rasgos morfo-estructurales.

SUMMARY: We make the commentary of four tropical species found at different states in SE of Spain, scarcely quoted in the Mediterranean Sea. This species are: Dipterosiphonia dendritica (Ag.) Schmitz in Engl. et Prantl, Liagora farinosa Lamouroux, Polystrata fosliei (Weber van Bosse) Denizot and Pocockiella variegata (Lamouroux) Papenfuss.

Como consecuencia de los estudios ficológicos que se vienen efectuando en los últimos años en el litoral murciano-almeriense (SE de la Península Ibérica) se ha determinado la presencia de cuatro especies poco citadas en la bibliografia algal del Mediterráneo y de un área de distribución tropical y pantropical. Se contribuye de esta forma a su corología y paralelamente se reafirma la existencia de un claro componente algal de afinidad termófila en este área, así como la existencia de ciertas particularidades anatómicas de estos ejemplares con relación a las descripciones clásicas de los mismos.

Dipterosiphonia dendritica (Ag.) Schmitz

MATERIAL ESTUDIADO: Murcia, Aguilas, 15-VI-84, J. Soto. (Phyc. $\mathrm{MGC}$ ).

ECOLOGIA: Epifito sobre Digenea simplex (Wulfen) Ag., en una comunidad infralitoral fotófila de ambiente calmo a $-1 \mathrm{~m}$.

DISTRIBUCION MUNDIAL: Según nuestros datos y los de Taylor (1960), Seoane (1965), Diaz-Piferrer (1969,1970), CordeiroMarino (1978), Gil-Rodríguez \& Afonso (1980), Verlaque (1981) y Weischer (1982), la distribución geográfica es la siguiente: Mediterráneo (España: Murcia; Córcega); Atlántico (España: Cádiz; Islas Canarias; Salvajes; Madeiras; Carolina del Norte; Islas del Caribe; Antillas Venezolanas; Colombia; Argentina: Patagonia; Oriente brasileño); Indico (Somalia y Tanzania); Pacífico (Isla de Pascua, Australia). A raíz de esta distribución mundial la especie debe ser considerada pantropical. 


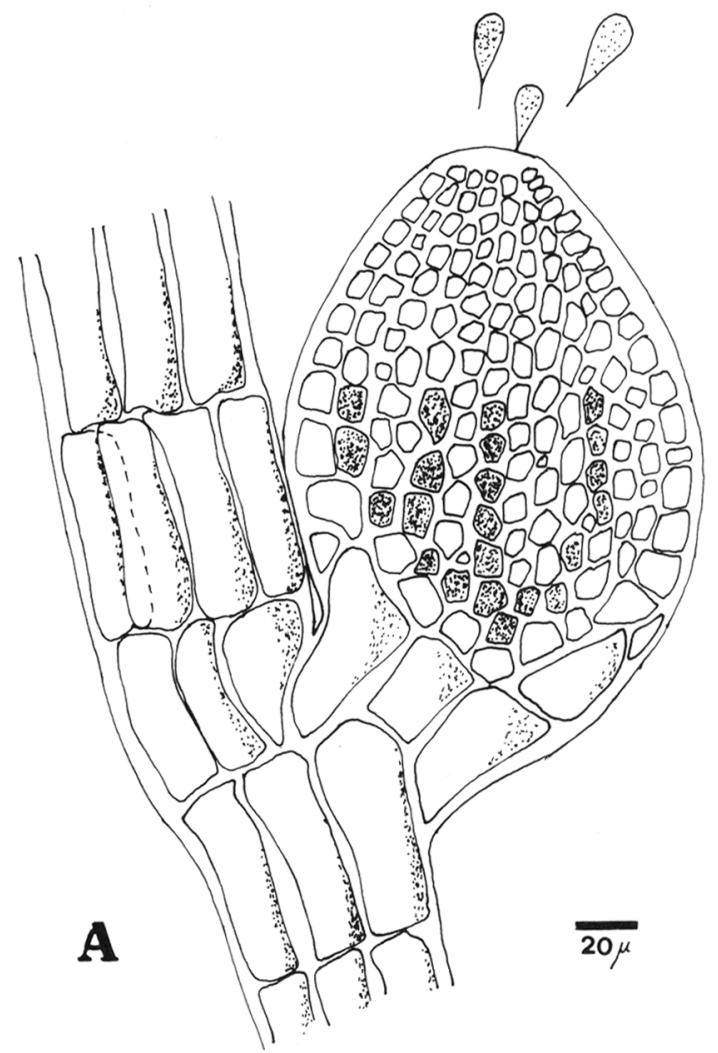

J. SOTO \& F. CONDE

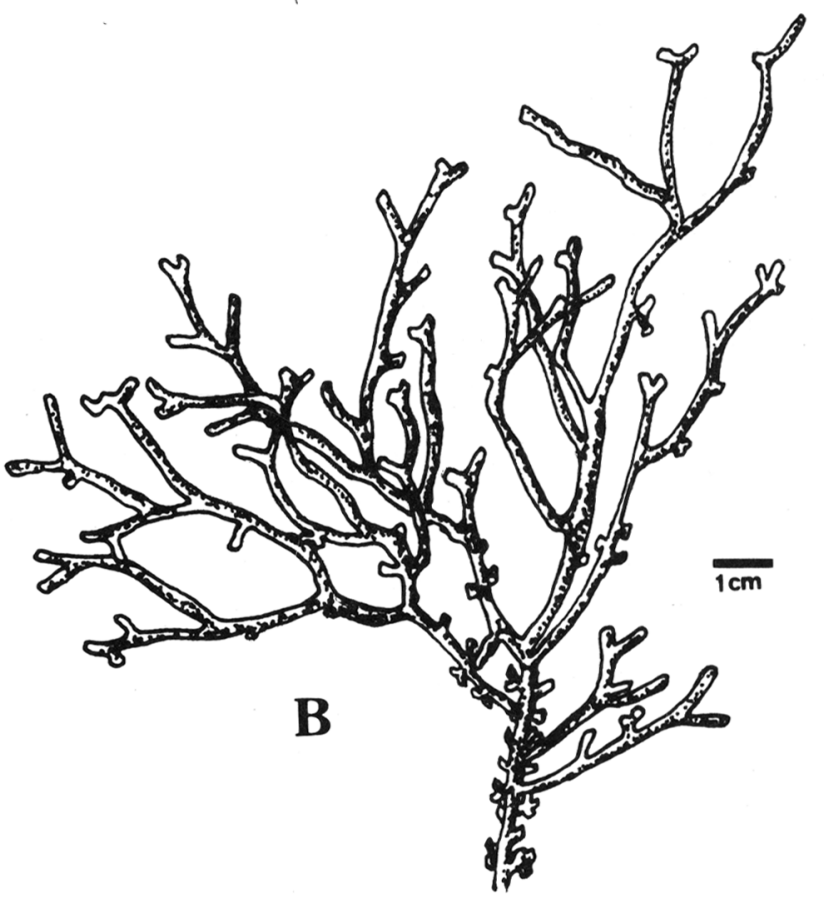

Fiqura 1: A.- Cistocarpo de Dipterosiphonia dentritica. B.- Hábito de Liagora farinosa. 
OBSERVACIONES: Los ejemplares se presentan fructificados con cistocarpos (fig. 1: A). De forma general se adaptan, con restricciones, a la descripción de Börgesen (1925-30), pués la alternancia de ramificación distica cada dos segmentos sucesivos no se aprecia siempre. Con la iconografía de Verlaque (1981) se diferencian por presentar seis células pericentrales en lugar de cinco.

\section{Liagora farinosa Lamouroux}

MATERIAL ESTUdiAd0: Murcia: Aguilas, IX-84, 10-VIII-85 y Calarreona, desde VII hasta XI de 1986, J. Soto. (Phyc. MGC). ECOLOGIA: Forma parte de comunidades fotófilas de ambiente calmo entre -0.5 y $-5 \mathrm{~m}$ pudiendo constituir en éstas una facies homogénea.

DISTRIBUCION MUNDIAL: Es propio este taxon de un claro componente tropical, encontrado en el Mediterráneo oriental antes de 1a abertura del Canal de Suez (Rayss in Mayhoub, 1976) por lo que la única posible vía de entrada en el Mediterráneo es el Estrecho de Gibraltar, o bien puede tratarse de una especie relictica en este mar que prolifera en sus áreas más termófilas.

Según nuestros propios datos y los de Börgesen (1915-1920), Taylor (1960), Diaz-Piferrer (1963, 1964, 1969), Papenfuss (1968), Giaccone (1969, 1972-73), Jaasund (1976), Mayhoub (1976), Gi1-Rodríguez \& Afonso (1980), Meñez \& Calumpong (1981) y Lawson \& John (1982), su distribución geográfica es la que sigue a continuación: Mediterráneo (España: Murcia; Italia: Ischia y Sicilia: Islas Eolias; Siria); Atlántico (Islas Canarias; Africa Occidental; Carolina del Norte y Florida; Islas del Caribe; Brasil); Mar Rojo (Ghardaqa, proximidades de Suez, Tor); Indico (Tanzania); Pacífico (Australia; Nueva Guinea; Filipinas).

OBSERVACIONES: Nuestros ejemplares son mayores que 1 a descripción que de esta especie hace Taylor (1960), alcanzando de 15 a $20 \mathrm{~cm}$ de longitud, y aún teniendo el modelo de ramificación dicótomo, presenta muchas proliferaciones laterales cortas (fig. 1B).

Polystrata fosliei (Weber van Bosse) Denizot

MATERIAL ESTUdiAdo: Almería: Villaricos, 8-XII-84,J. Soto (Phyc $\mathrm{MGC}$ ) .

ECOLOGIA: Infralitoral superior esciáfilo y batido $(-0.5 \mathrm{~m})$.

DISTRIBUCION MUNDIAL: Es una especie poco conocida, desde un punto de vista corológico, ya que según nuestros datos y los de Denizot (1968), Segonzac (1969) y Verlaque (1981) su distribución es hasta ahora en las aguas cálidas del Mediterráneo (España: Almería; Córcega); Atlántico (Islas de Cabo Verde y Haiti: Isla Tortuga) y Pacifico (Indonesia). Probablemente sea un taxon tropical de corología mucho más amplia.

OBSERVACIONES: Los ejemplares encontrados se ajustan totalmente a la descripción dada por Denizot (1968).

Pocockie1la variegata (Lamouroux) Papenfus

MATERIAL ESTUdiAdo: Murcia: Aguilas, VII a XII de 1984; Almeria: Villaricos, I, II y $x$ de 1984, J. Soto (Phyc. MGC). ECOLOGIA: Se encontró recubriendo un talo de Lythophyllum 


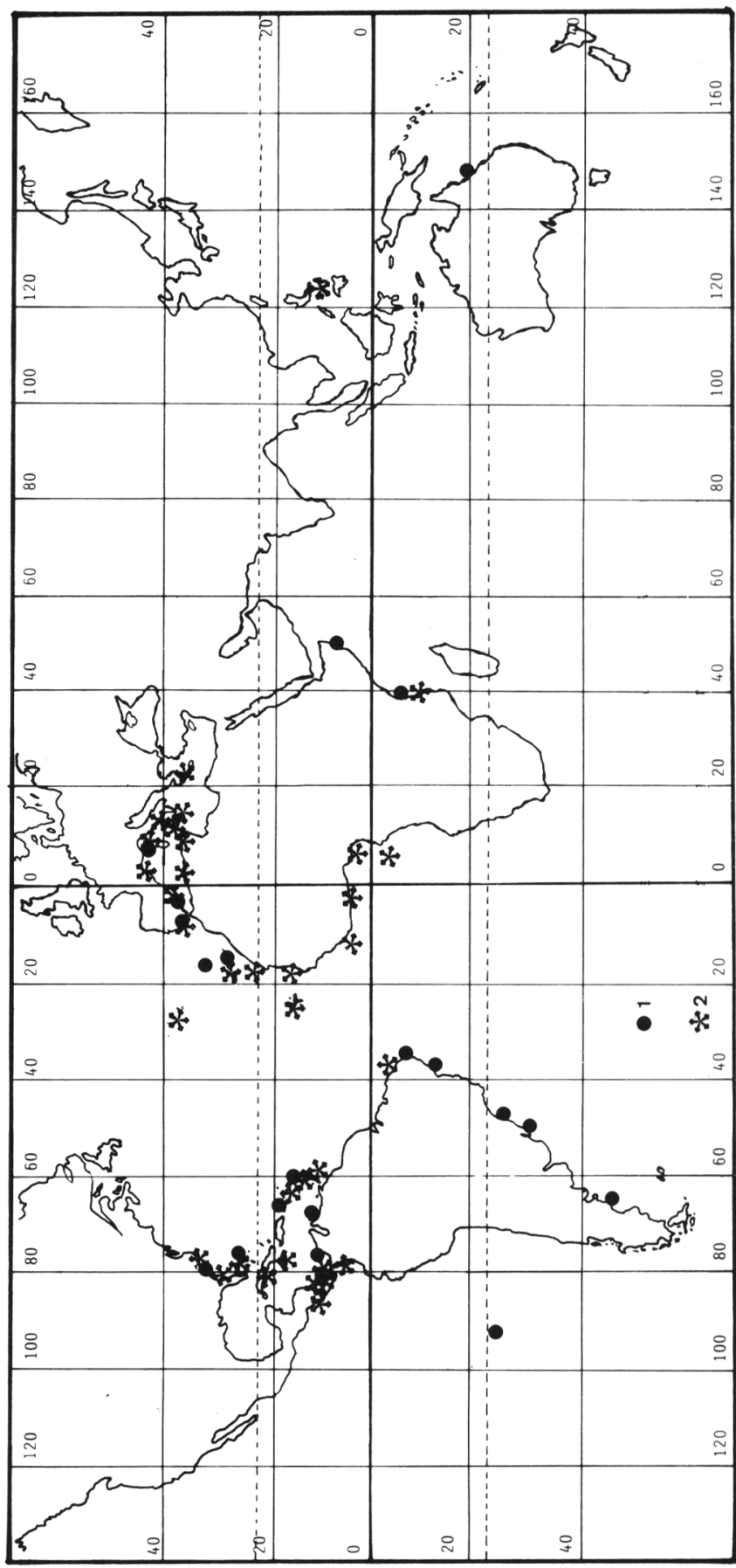

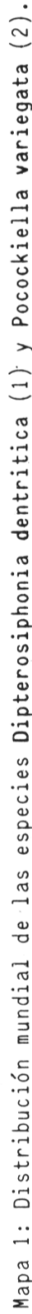




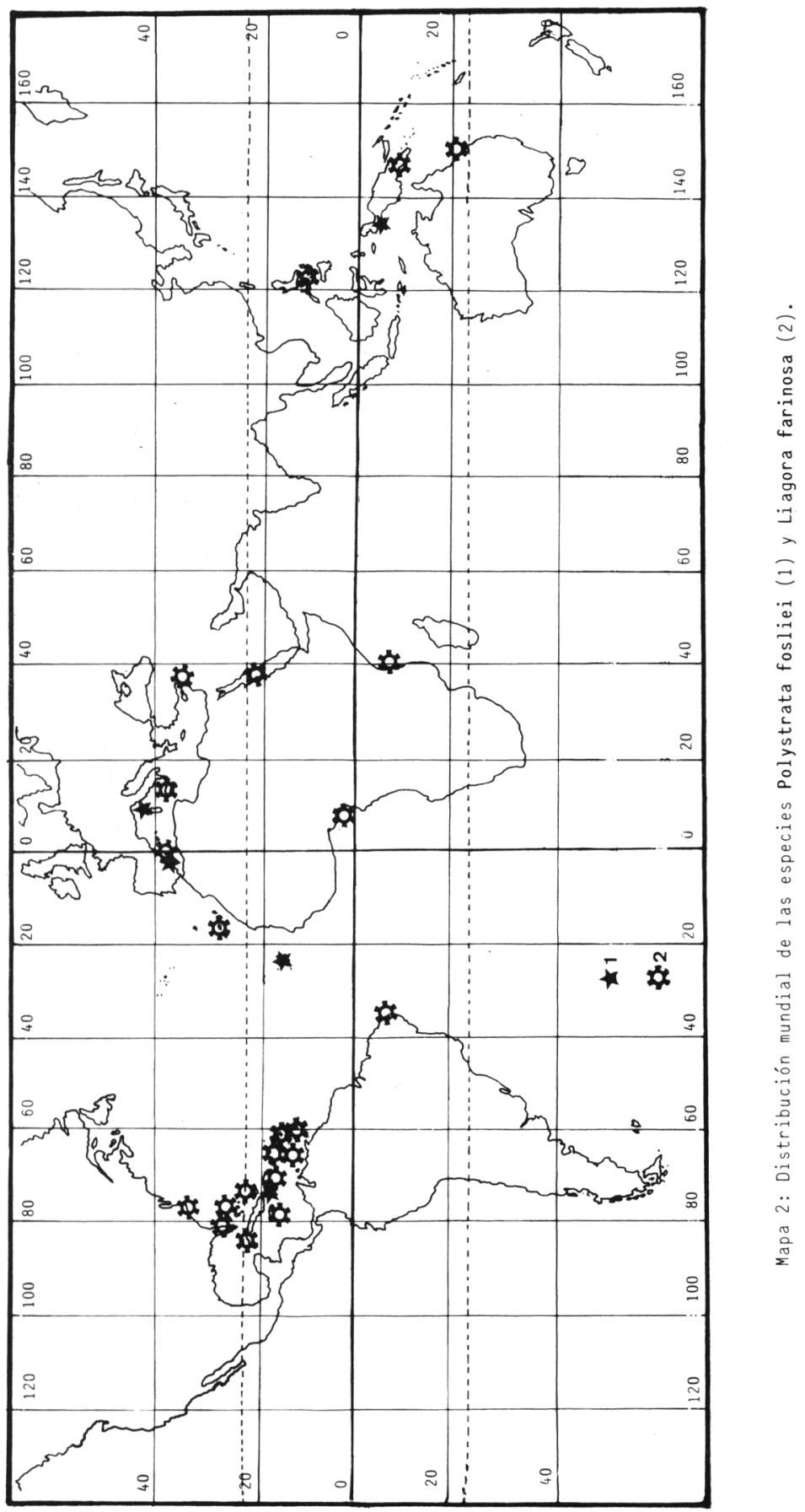


incrustans Phil. a poca profundidad en un nicho hemiesciáfilo. DISTRIBUCION GEOGRAFICA: Geográficamente, este alga se extiende por zonas de mares cálidos, por lo que está bien representada en el Mediterráneo y entre los trópicos. Según nuestros datos y los de Hame1 (1931-1939), Taylor (1960), Dawson (1962), Díaz-Piferrer (1963, 1964, 1969), Boudouresque \& Boudouresque (1969) Boudouresque (1973), Belsher, Augier, Boudouresque \& Coppejans (1976), Boudouresque \& Cinel1i (1976), Jaasund (1976), Boudouresque \& Perret (1977), Cine11i, Fresi, Mazze11a \& Ponticel1i (1979), Cormaci \& Furnari (1979), Gil-Rodríguez \& Afonso (1980), Meñez \& Calumpong (1981), Lawson \& John (1982), Schneter \& Bula Meyer (1982), Weischer (1982) y Llimona \& a 1 . (1985), su distribución corológica es la siguiente: Mediterráneo (España; Francia; Italia: Canal de Sicilia, Islas de Linosa; Argelia; Tunizia-Norte?; Córcega y Creta); Atlántico (España: Cádiz, Canarias; Salvajes; Madeiras; Azores; Cabo Verde; desde el Sahara a Gabón; Carolina del Norte y Florida; Islas del Caribe; Costa Rica; Panamá; Colombia y Brasil); Indico (Tanzania); Pacífico (Nicaragua, Colombia y Filipinas). OBSERVACIONES: Los ejemplares recolectados son adherentes de porte laminar y borde flabelado. Estructuralmente coinciden con las descripciones de múltiples autores.

\section{CONCLUSIONES}

1 o La distribución de estas cuatro especies permite constatar, de forma amplia, que se encuentran entre los Trópicos de Cáncer y Capricornio, excepto Dipterosiphonia dentritica y Pocockiella variegata, aparentemente más cosmopolitas, no obstante, ambas incluidas dentro de regiones fitogeográficas cálidas (Mapa 1 y 2 ).

2ㅇ La localización de estas especies en el SE peninsular es atribuible al carácter acentuadamente cálido de sus aguas costeras que casi un $75 \%$ de los días del año se encuentran por encima de 1 os $17^{\circ} \mathrm{C}$, con minimas de $14.5^{\circ} \mathrm{C}$ y máximas de $27^{\circ} \mathrm{C}$ además del hecho de su proximidad con el Atlántico cálido (Hoek, 1984), sólo separada de él por el Estrecho de Gibraltar.

3o La distribución de estos táxones es rara en el Mediterráneo, excepto la de Pocockie11a variegata que es amplia, especialmente en el Mediterráneo occidental.

4을 Excluida Dipterosiphonia dentritica (Seoane, 1965) y Pocockiella variegata (Hame1, 1931-39, Llimona et a1., 1985) se puede afirmar, que en la Península Ibérica estas cuatro especies han sido muy poco citadas (cf. Ga11ardo et a1., 1985).

\section{BंIBLIOGRAFIA}

BELSHER, T., AUGIER, H., BOUDOURESQUE, C.F. \& COPPEJANS, E. -1976Inventaire des algues marines benthiques de la rade et des iles d'Hyères (Méditerranée, France). Trav. sci. Parc. nation. Port-Cros, $2: 39-89$. 
BORGESEN, F. -1915/20- The marine algae of the Danish West Indies. Rhodophyceae. Dansk Bot. Arkiv. 504 pp. Copenhagen.

BORGESEN, F. -1925/30- Marine algae from the Canary Islands, especially from Teneriffe and Gran Canaria. II Phaeophyceae (1926). III Rhodophyceae. 1 Bangiales and Nemalionales (1927). Dett. Kgl. Danske Videns. Selsk., Biol. Medd., $112+97$ pp. Copenhagen.

BOUDOURESQUE, C.F. -1973- Recherches de bionomie analytique, estructurale et expérimentale sur les peuplements benthiques sciaphiles de Méditerranée occidentale (fraction algale). Les peuplements sciaphiles de mode relativement calme sur substrats durs. Bull. Mus. Hist. Nat. Marseille, 33:147-225.

BOUDOURESQUE, C.F. \& BOUDOURESQUE, E. -1969- Contribution a la flore des algues marines de l'Algerie. Bull. Mus. Hist. Nat. Marseille, 29:129-136.

BOUDOURESQUE, C.F. \& CINELLI, F. -1976- Le peuplement algal des biotypes sciaphiles superficiels de mode battu en Méditerranée occidentale. Tirés-à-part des Pubbl. Staz. Zool. Napoli, 40:433-459.

BOUDOURESQUE, C.F. \& PERRET, M. -1977- Inventaire de la flore marine de Corse (Méditerranée): Rhodophyceae, Phaeophyceae, Chlorophyceae et Bryopsidophyceae. Bibliotheca Phycolog., vol. 25, 171 pp. J. Cramer. Vaduz.

CINELLI, F., FRESI, E., MAZELLA, L \& PONTICELLI, M.P. -1979- Deep algal vegetation of the western Mediterranean. Giorn. Bot. Ital., 113:173-188.

CORDEIRO-MARINO, M. -1978- Rodofíceas bentônicas marinhas do estado de Santa Catarina. Rickia, 7:1-243.

CORMACI,M. \& FURNARI, G. -1979- Flora algale marina della Sicilia Orientale: "Rhodophyceae, Phaeophyceae e Chlorophyceae". Bull. Soc. Bot. Italiana, 11 (2):221-250.

DAWSON, E.Y. -1962- Additions to the marine flora of Costa Rica and Nicaragua, Pacific Naturalist, 3 (13):375-395.

DENIZOT, M. -1968- Les algues floridées encroutantes (à l'exception des Corallinacées). Lab. Cryp. Mus. Hist. Nat. Paris, 310 p.

DIAZ-PIFERRER, M. -1963- Adiciones a la flora marina de Puerto Rico. Carib. J. Sci., $3(4): 215-235$.

DIAZ-PIFERRER, M. -1964- Adiciones a la flora marina de las Antillas Holandesas, Curaçao y Bonaire. Carib. J. Sci., 4 (4):513-543.

DIAZ-PIFERRER, M. -1969- Distribution of the marine benthic flora of the Caribbean Sea. Carib. J. Sci., 9 (3-4):151-178.

DIAZ-PIFERRER, M. -1970- Adiciones a la flora marina de Venezuela. Carib. J. Sci., 10 (3-4):159-198.

GAllaRdo, T., GOMEZ-GARRETA, A., RIBERA, M.A. ALVAREZ, M. \& CONDE, F. 1985- A preliminary checklist of Iberian benthic marine algae. Ed. Real Jardín Botánico de Madrid, $83 \mathrm{pp}$.

GIACCONE, G. -1969- Raccolte di fitobenthos sulla banchina continentale italiana. Estratto da: Giorn. Bot. Ital., 103:485-514.

GIACCONE, G. -1972/73- Elementi di Botanica Marina. Parte seconda. Chiavi di determinazione per le alghe e le angiosperme marine del Mediterraneo. Instituto di Botanica dell' Università degli Studi di Trieste, $358 \mathrm{pp}$.

GIL-RODRIGUEZ, M.C. \& AFONSO, J. -1980- Catálogo de las algas marinas bentónicas (Cyanophyta, Chlorophyta, Phaeophyta y Rhodophyta) para el Archipiélago Canario. Aula de cultura de Tenerife, $66 \mathrm{pp}$.

HAMEL, G. -1931/39- Phéophycées de France. XLVII +431 pp., 60 fig., 10 pl. Paris.

HOEK, C. VAN DEN -1984- World-wide latitudinal and longitudinal seaweed distribution patterns and their possibles causes, as illustrated by the distribution of Rhodophytan genera. Helgolländer Meeresunters, $38: 227-257$. 
JAASUND, E. -1976- Intertidal Seaweeds in Tanzania. University of Tromso, $160 \mathrm{pp}$.

LAWSON, G.W. \& JOHN; D.M. -1982- The marine algae and coastal environment of Tropical West Africa, $455 \mathrm{pp}$. J. Cramer. Vaduz.

LLIMONA, X. \& al. -1985- Historia natural dels Paisos Catalans. Plantes inferiors. Enciclopedia Catalana, vol. 4, 558 pp. Barcelona.

MAYHOUB, H. -1976- Recherches sur la vegetation marine de la côte Syrienne. Etude experimentale sur la morphogenese et le développement de quelques espèces peu connues. Thèse de doctorat d'ètat. Université de Caen. $286 \mathrm{pp} ., 15 \mathrm{pl}$.

MEÑEZ,E.G. \& CALUMPONG, H.P. -1981- Phycological results of the Smithsonian institution -Philippines expeditions of 1978 and 1979 in Central Visayas, Philippines. Proceeding of the Fourth International Coral Reef Symposium, Manila. vol. 2. p. 379-384.

PAPENFUSS, G.F. -1968- A history, catalogue, and bibliography of Red Sea benthic algae. Israel Journ. of Botany, 17:1-118.

SEGONZAC, G. -1969- Présence de Polystrata fosliei dans la mer des Caraībes. Rev. algol., 9:359-360.

SEOANE, J.A. -1965- Estudios sobre las algas bentónicas en la costa sur de la Península Ibérica (1itoral de Cádiz). Inv. Pesq., 29:3-216.

SCHNETER, R. \& BULA MEYER, G. -1982- Marine algen der Pazifikküste von Kolumbien. Chlorophyceae, Phaeophyceae, Rhodophyceae. Bibliotheca Phycologica. Band 60. 287 pp. J. Cramer. Vaduz.

TAYLOR, W.R. -1960- Marine algae of the eastern tropical and subtropical coasts of the Americas. Ann. Arbor. Univ. Michigan Press., 21:1-870.

VERLAQUE, M. -1981 * Contribution à la flore des algues marines de Méditerranée: Espèces nouvelles pour la Méditerranée occidentale. Bot. Mar., 24:559-568.

WEISCHER, F.C. -1982- Marine algae from Ilhéu de Fora (Salvage Islands). (Cancap-project contributions no. 13). Boletim do Museu Municipal do Funchal, 34:23-34. 